\title{
The Dielectric Wedge Antenna
}

\author{
Alexander G. Yarovoy, Member, IEEE, Alexander D. Schukin, Igor V. Kaploun, and Leo P. Ligthart, Fellow, IEEE
}

\begin{abstract}
A new modification of the TEM-horn antenna has been developed for ground-penetrating radar (GPR) applications. The antenna is based on a dielectric wedge, and thus, was named the dielectric wedge antenna. Tapering of the metal flairs has been used to match the antenna to the ground and to reduce the late-time ringing. The finite-difference time-domain model of the antenna has been created to investigate physical processes within the antenna and to optimize its performance. The dielectric wedge antenna has shown a superior (in comparison with the TEM-horn) performance by radiation in different types of the ground and has been used in a novel antenna configuration within a GPR system, which is dedicated for landmine detection.
\end{abstract}

Index Terms-Finite-difference time-domain (FDTD), ground penetrating radar, TEM-horn antenna, transient antenna, ultra-wideband antenna.

\section{INTRODUCTION}

A NTENNAS for impulse ground penetrating radar (GPR) are impulse radiating antennas that should satisfy a number of specific demands. First, the antennas should radiate pulses with given properties into the ground and receive pulses scattered from subsurface objects [1]. As a rule, the ground situated in the near zone of the antenna has a large impact on the antenna performance [2] and [3]. However, an antenna performance that is stable for different ground types and different antenna elevations above the ground is desirable. Because GPR antennas are used in a short-range high-resolution radar system and the radiated electromagnetic (EM) field must penetrate as deep as possible in a lossy medium, they should operate at reasonably low frequencies (typically below two gigahertz) and cover a wide-frequency band (typically more than several hundreds megahertz). Therefore, the antennas should be ultra wideband [4]. Because coupling in the transmit-receive antenna pair can obscure reflections from shallow buried targets and can substantially limit the dynamic range of the whole GPR system, high transmit-receive ( $\mathrm{Tx}-\mathrm{Rx})$ antenna isolation is also an important characteristic for GPR antennas. Finally, antennas used in video-impulse radars should also have linear phase characteristics and constant polarization [1]. Such a combination of sometimes controversial demands makes the development of GPR antennas an extremely difficult task. Until now, only a few GPR antennas have been developed [5],

Manuscript received February 21, 2001; revised August 30, 2001. This work was supported in part by the Dutch Technology Foundation STW, by the Applied Science Division of NWO, and by the Technology Program of the Ministry of Economic Affairs of The Netherlands.

A. G. Yarovoy and L. P. Ligthart are with the International Research Centre for Telecommunications-Transmission and Radar, Department of Information Technology and Systems, Delft Universtiy of Technology, Delft, The Netherlands (e-mail: a.yarovoy@its.tudelft.nl).

A. D. Schukin and I. V. Kaploun are with the Academician A. L. Mints Radiotechnical Institute (RTI), 125083 Moscow, Russia.

Digital Object Identifier 10.1109/TAP.2002.803968
[6] and the simplest ones, like the resistively loaded dipole and the resistively loaded bow tie, remain the most frequently used GPR antennas. A recent demand for high-resolution GPR systems for motorway or runway inspection [7], unexploded ordinance, and landmine detection [8] has become a driving force in the development of more sophisticated GPR antennas.

This paper introduces a new GPR antenna: the dielectric wedge antenna. This antenna is based on a dielectric wedge and has a number of similarities with dielectric-filled TEM horns [9], and [10]. The main constructional difference of the proposed antenna from a dielectric-filled TEM horn is the tapering of antenna flairs. An additional difference, is that the dielectric base of this antenna does not have a pyramidal shape as in TEM horns [9], [10], but a wedge shape. The tapering profile and the size of the dielectric wedge have been optimized to improve antenna matching to the ground and to reduce antenna late-time ringing. Due to this design it became possible to increase the antenna efficiency (in comparison to that of an ordinary TEM horn), to improve the Tx-Rx antenna isolation and to decrease the antenna size considerably in all three dimensions. Antenna design considerations are presented in Section II. The theoretical simulation of the antenna is discussed in Section III, while Section IV describes the experimental verification. Section $\mathrm{V}$ discusses antenna features and possible applications of the antenna.

\section{DESIGN CONSIDERATIONS}

One of the best known antennas for the radiation of short pulses is the TEM horn. This antenna can radiate signals over an ultra-wide frequency band and has a linear phase characteristic over this band. Although the radiated pulse does not widen (in comparison with the feeding pulse), a part of the propagated inside the horn pulse is reflected from the antenna aperture back into the antenna and is then re-radiated again from the feed point (late-time antenna ringing). This re-radiation increases when the antenna is pointed to the ground and elevated only a few centimeters above it. For a short-range radar system (like GPR) this drawback of TEM horns causes essential problems because the reflection from the aperture can mask a target return. To avoid this re-radiation, different kinds of resistive loading schemes were proposed (see e.g., [11]). However, as resistive loading absorbs energy of the radiated pulse, it decreases the antenna efficiency. Other disadvantages of conventional TEM horns are their high sensitivity to external electromagnetic interference (EMI) and the strong coupling between two such antennas used in a radar system with separate Tx and Rx antennas. This coupling can obscure reflections from shallow buried targets and can substantially limit the dynamic range of the whole GPR system. When the antenna system is placed on the ground, the coupling becomes even worse due to additional ground-wave 


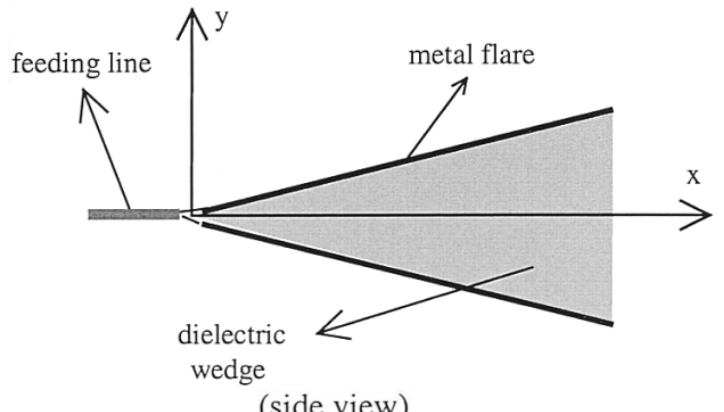

(side view)

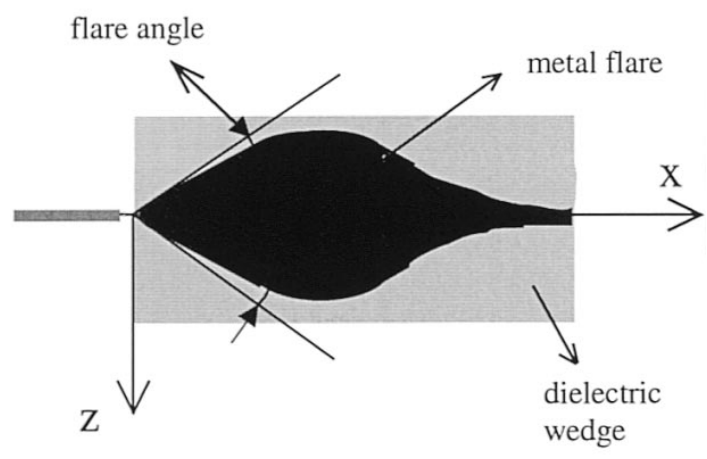

(top view)

Fig. 1. A dielectric wedge antenna with its coordinate system.

coupling. Finally, the large physical dimensions of the TEM horn (typically its size equals three times the duration of the pulse multiplied with the velocity of light) cause an additional problem for application in GPR systems. To overcome these disadvantages different researchers have attempted to fill a TEM horn with dielectric. Different antenna designs have been proposed by various authors e.g., [9] and [10]. These designs use a dielectric pyramid as a base for the antennas. The metal flairs of these antennas have a nontapered triangular shape. The antenna presented in this article has tapered metal flairs and is based on a dielectric wedge. The schematic drawing of this antenna is presented in Fig. 1.

A prototype of the dielectric wedge antenna is the tapered TEM horn [12]. The latter was developed based on similar ideas as the TWIT antenna [13], but the tapering profile of the metal flairs differ. We decided to improve the performance of this antenna (i.e., to improve antenna matching to the ground, to reduce the late-time ringing, to decrease the coupling and the sensitivity to external EMI) by placing it on a dielectric wedge. A wedge geometry was chosen instead of a pyramidal one because of the large value $\left(108^{\circ}\right)$ of the flair angle that is required for antenna matching to the feeding line. In a pyramidal geometry the pyramid vertex angle should be equal to the flair angle and a pyramid with the vertex angle of $108^{\circ}$ would have much larger dimensions and weight than the wedge.

We carried out a number of finite-difference time-domain (FDTD) simulations of the wedge itself to investigate the field structure within the wedge, to check for internal resonances of the wedge and to find the optimal size and wedge inclination angle of the wedge for a given dielectric permittivity (the FDTD model is described in detail in the next Chapter). The wedge has been excited with a current source in the middle of its top edge. We found that the transient current excites two transient waves: one which propagates within the wedge and another that propagates outside. The former was named the wedge wave and the latter the air wave. The air wave has the same waveform as the excitation current and arrives earlier than the other signals at any observation point outside the wedge. The wedge wave, while propagating within the wedge, also has the same waveform as the excitation current. The maximal wave amplitude has been observed propagating along the sidewalls of the wedge. A part of this wave energy (of about $0.3 \%$ ) bounces back from the aperture of the wedge, returns to the top edge, bounces once more and re-radiates from the wedge, causing the late-time ringing. The electric field radiated in the forward direction in the far zone of the wedge consists of contributions of the air wave and of first-, second- and higher-order pulses radiated by the wedge wave (Fig. 2).

To minimize the reflections from the antenna aperture, we chose the dielectric permittivity of the wedge as close as possible to the dielectric permittivity of a typical ground. For the antenna, which should be matched to a dry sandy soil, a value 3.5 has been selected. By varying the wedge size (for the given dielectric permittivity) it is possible to vary the delay time between the arrival time of the air wave and that of the wedge wave. In such a way, the optimal combination of the wedge size and permittivity can be found so that the second part of the air wave arrives simultaneously with the first part of the wedge wave, and they both add in magnitude. As a result, for a dielectric permittivity of 3.5 an optimal wedge height of $25.25 \mathrm{~cm}$ was found with the aperture of $16 \times 16 \mathrm{~cm}$. With this geometry we achieved the minimal widening of the radiated pulse together with its maximal magnitude.

After determining the optimal size (for the given dielectric permittivity) of the wedge, we investigated the shape of antenna's metal flairs. The antenna has been considered as a microstrip transmission line with slowly varying parameters. The cross section of the dielectric substrate and the width of the metal strip varies with the microstrip line length. The characteristic impedance of the microstrip line can be calculated from [14]

$$
\begin{gathered}
Z_{0}=376,687 *(2 \sqrt{\varepsilon})^{-1} *\left\{\frac{W}{2 h}+0,441+0,082 \frac{(\varepsilon-1)}{\varepsilon^{2}}\right. \\
\left.+\left[\frac{(\varepsilon+1)}{2 \pi \varepsilon}\right] *\left[1,451+\ln \left(\frac{W}{2 h+0,94}\right)\right]\right\}^{-1}
\end{gathered}
$$

where $W$ is the width of the microstrip and $h$ is the thickness of the dielectric substrate and $\varepsilon$ is a relative dielectric permittivity of the substrate. A nonlinear optimization of the microstrip-line width has been carried out in order to achieve a smooth transition of the characteristic impedance of $50 \Omega$ to the impedance of a homogeneous space filled with the same dielectric as the wedge. The goal function was the minimal reflection from each 


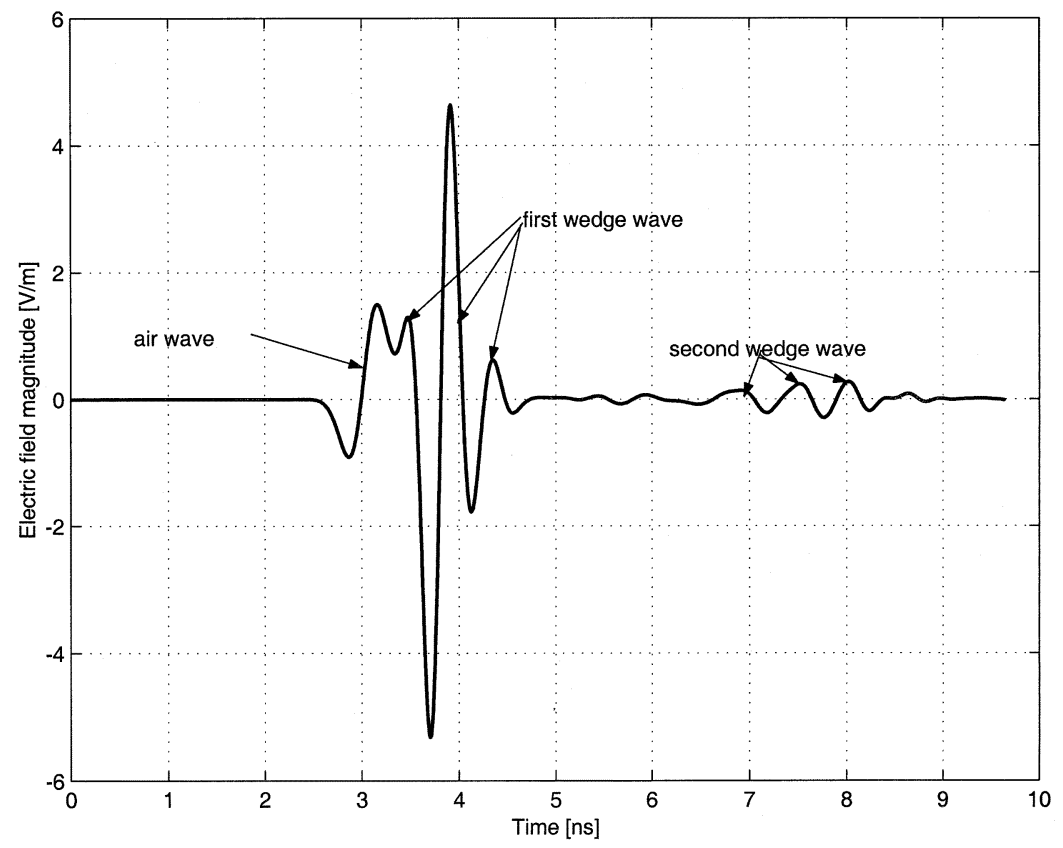

Fig. 2. Forward radiation from the wedge (the wedge height is $24.25 \mathrm{~cm}$; the foundation size is $16 \times 16 \mathrm{~cm}$; dielectric permittivity equals 4 ).

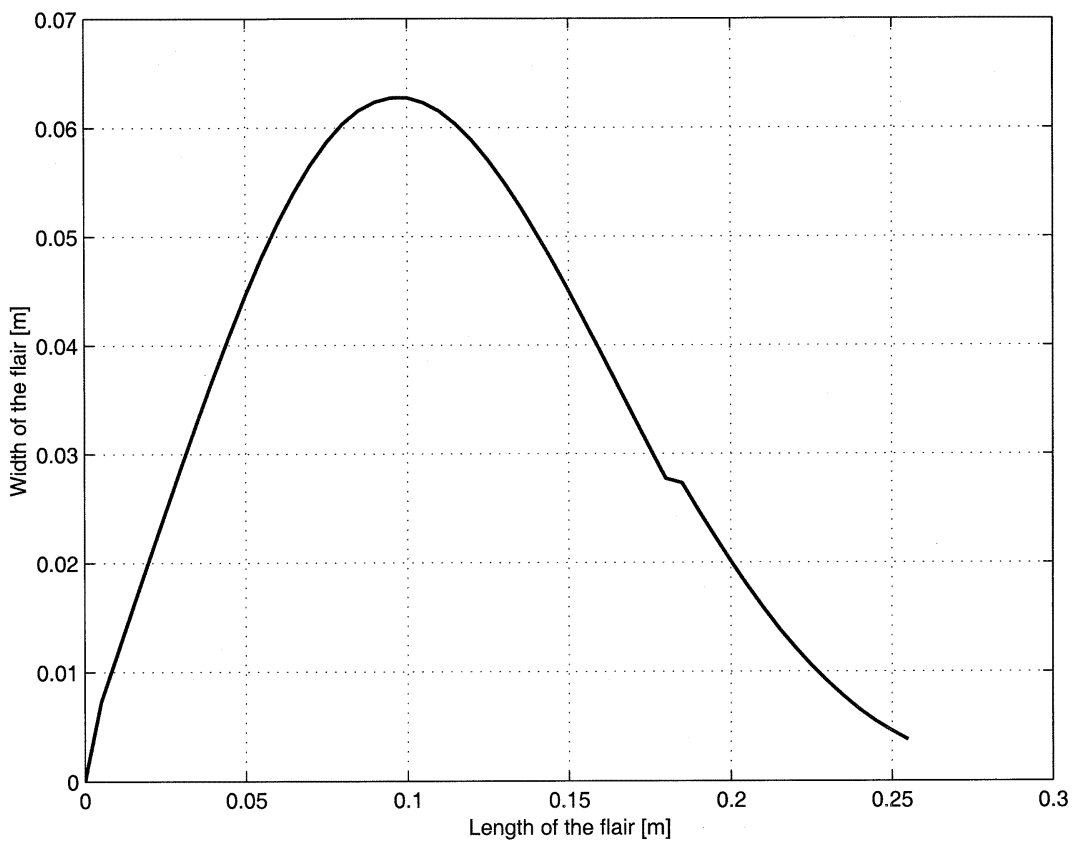

Fig. 3. Flair profile as calculated based on the microstrip-line theory.

cross section of the microstrip line. The resulting flair profile is presented in Fig. 3. For this profile minimal reflection from the microstrip line has been achieved.

\section{FDTD SIMULATION}

To justify the tapering profile and to analyze physical processes for transient radiation from a new antenna we developed a fully three-dimensional (3-D) FDTD model of this antenna. In order to use the FDTD model for antenna optimization, the model must meet two-major demands: high accuracy and fast computational time. The demand of minimal computational time is qualitatively different from the minimal computer storage demanded in a number of previous papers on FDTD simulations of transient antennas [15].

To reduce the size of computational volume and therefore, to decrease computational time, we used the antenna symmetry. In fact, only one quarter of the real antenna has been simulated. On the position of the planes of symmetry, electric $(y=0)$ and magnetic $(z=0)$ walls have been introduced. The electric (or magnetic) wall satisfies zero-value boundary conditions for the tangential component of electric (or magnetic) field. The computational volume is terminated by second-order Mur 


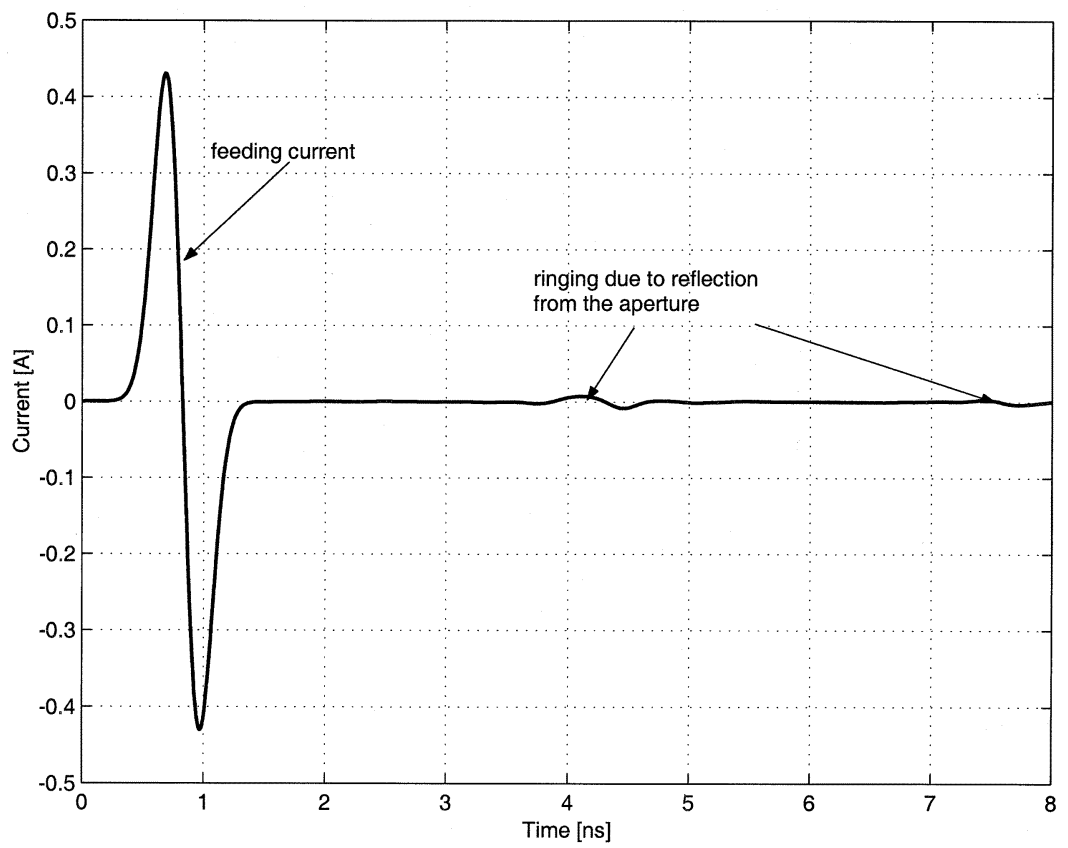

Fig. 4. Surface current on the metal flair as simulated by FDTD.

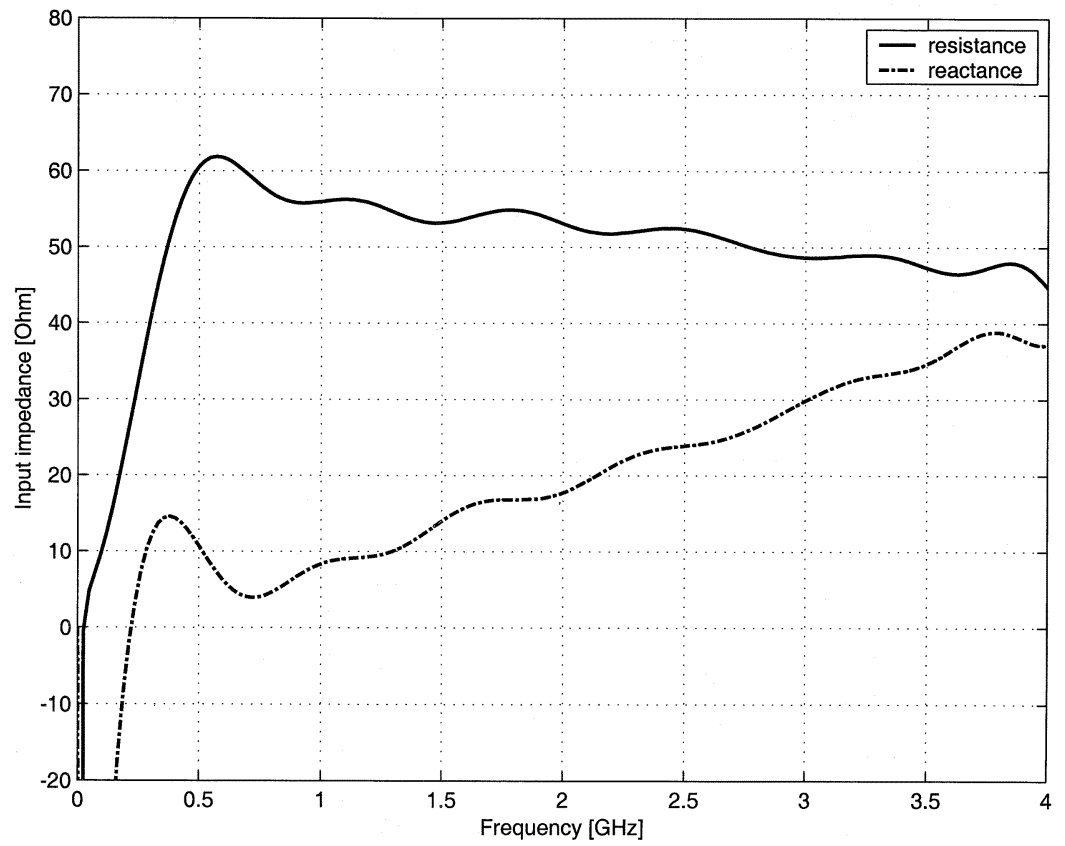

Fig. 5. Simulated input impedance of the antenna.

radiation boundary conditions [16] and by electric (magnetic) walls. During simulation of the antenna above the ground the computational volume outside the antenna is filled with two-homogeneous media, which represent air and ground. At places where the air-ground interface reaches the boundary conditions, simple first-order Mur absorbing boundary conditions have been used.

The FDTD model employs a homogeneous mesh where the computational volume is divided into rectangular parallelepiped of equal size. Despite the larger number of cells (in comparison with inhomogeneous meshes, e.g., the "ballooned" mesh in [15]), such a mesh leads to a computationally simpler and, there- fore, faster algorithm. The cell size has been chosen such that the maximum distance between adjacent corners of the staircase is much smaller than one wavelength (in the dielectric material) at the highest frequency of interest for the incident pulse. Particularly, for the wedge dielectric permittivity of around 4 and the monocycle pulse with duration of $0.8 \mathrm{~ns}$ the cell size of $2.5 \mathrm{~mm}$ has been selected. Such an approach makes it possible to avoid resonance phenomena due to the staircase approximation [15]. Varying parameters of the FDTD model (cell size, position of absorbing boundaries, etc.) we achieved that the computational error is substantially below $-40 \mathrm{~dB}$ within the observation time window. 


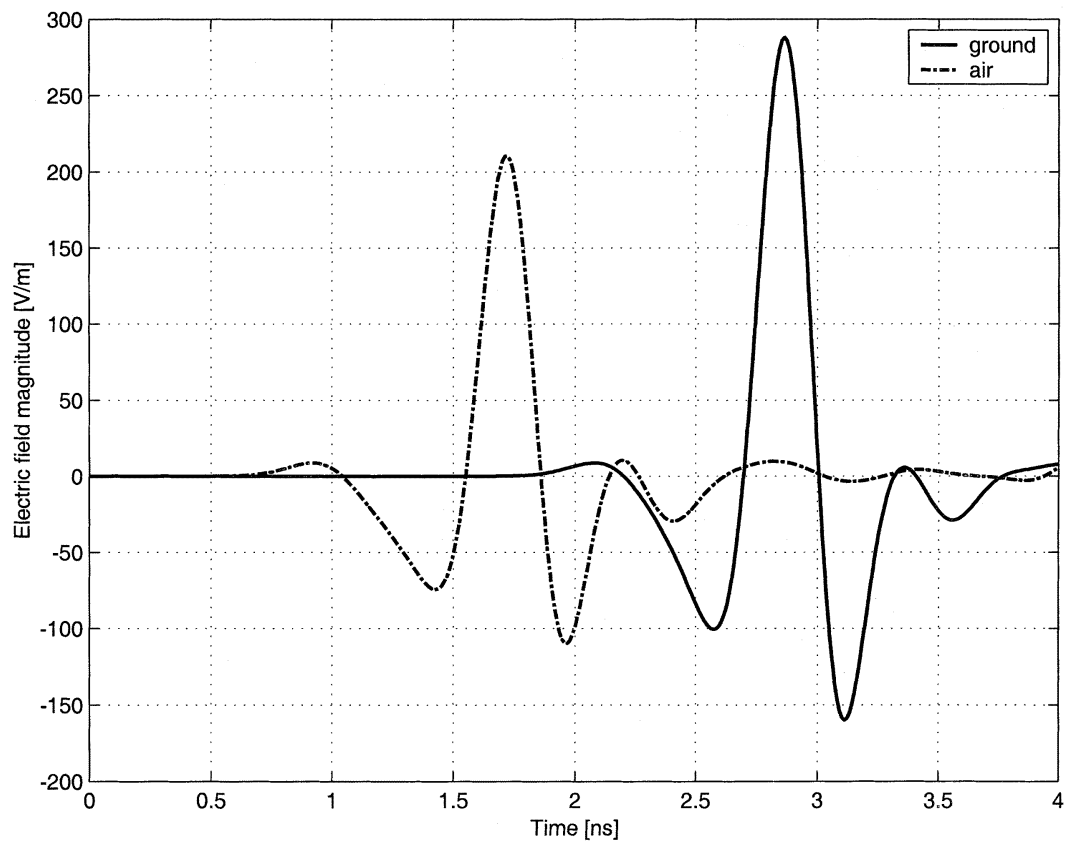

Fig. 6. Simulated radiation along the main axis into the ground (solid line) and into the air (dashed line) at the distance of $39 \mathrm{~cm}$ from the antenna aperture. Dielectric permittivity of the ground equals 3.5 and the ground conductivity equals $0.005 \mathrm{~S} / \mathrm{m}$.

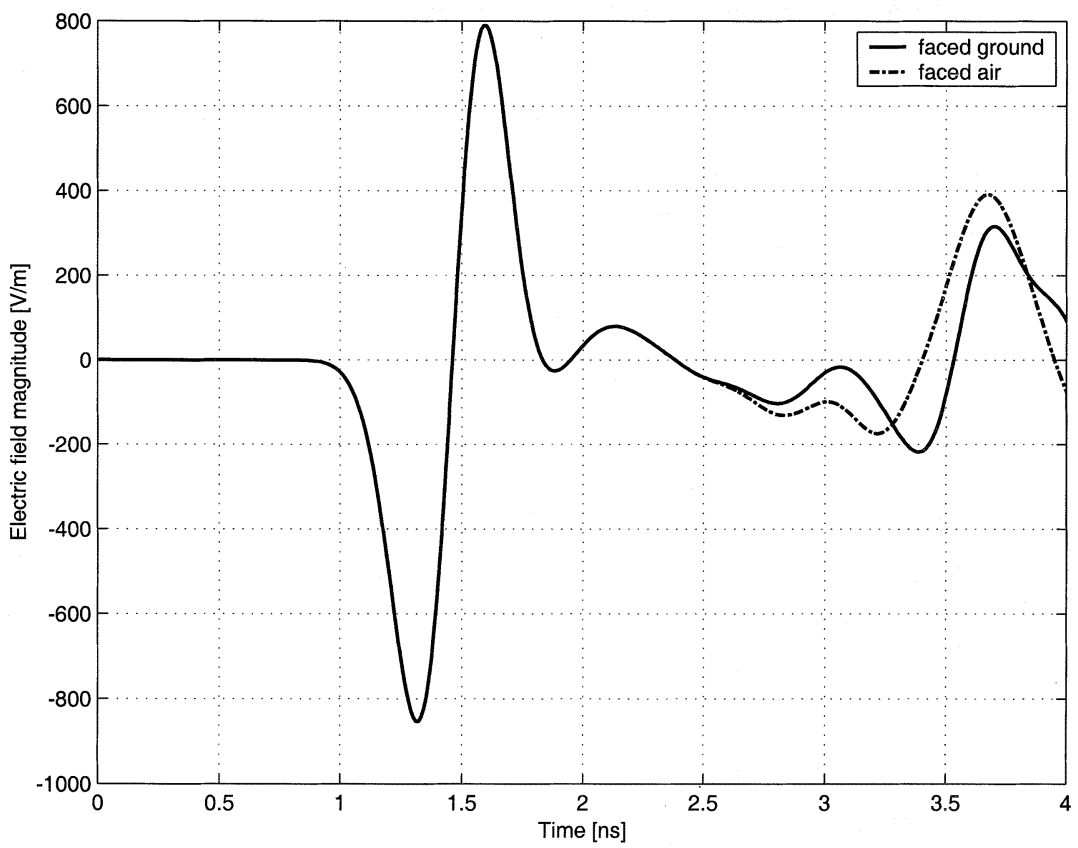

Fig. 7. Electric field on the main axis inside the antenna at the distance of $9.5 \mathrm{~cm}$ from the apex; aperture facing the ground (solid line) and the air (dashed line).

During simulations, the antenna was fed by a current source placed in the feed point of the antenna between the metal flair and the electrical wall at $y=0$. The magnitude of the current is homogeneous along the source and varies in time as a differentiated Gaussian pulse of the form

$$
V(t)=\frac{V_{0}\left(\frac{t}{\tau}-4\right) \exp \left(-\left(\frac{t}{\tau}-4\right)^{2}+\frac{1}{2}\right)}{\sqrt{2}}
$$

where $V_{0}$ is the peak-to-peak magnitude of the feeding pulse, which is assumed to be equal to $1 \mathrm{~V}$ and $\tau$ is the pulse width.
The pulse centre has been delayed by four pulse widths to avoid the usage of negative times. The influence of the feeding line has been modeled by a lumped $50 \Omega$-resistor placed in parallel with the feeding current.

From FDTD simulations of the complete antenna, we found that like in the wedge the transient source excites two waves in the antenna: the air wave and the wedge wave. The wedge wave is definitely not a TEM wave, so the microstrip-line simulation of the antenna is not fully adequate. However, the flair profile calculated on the basis of the microstrip-line theory causes no reflections from the internal cross sections of the antenna. The 


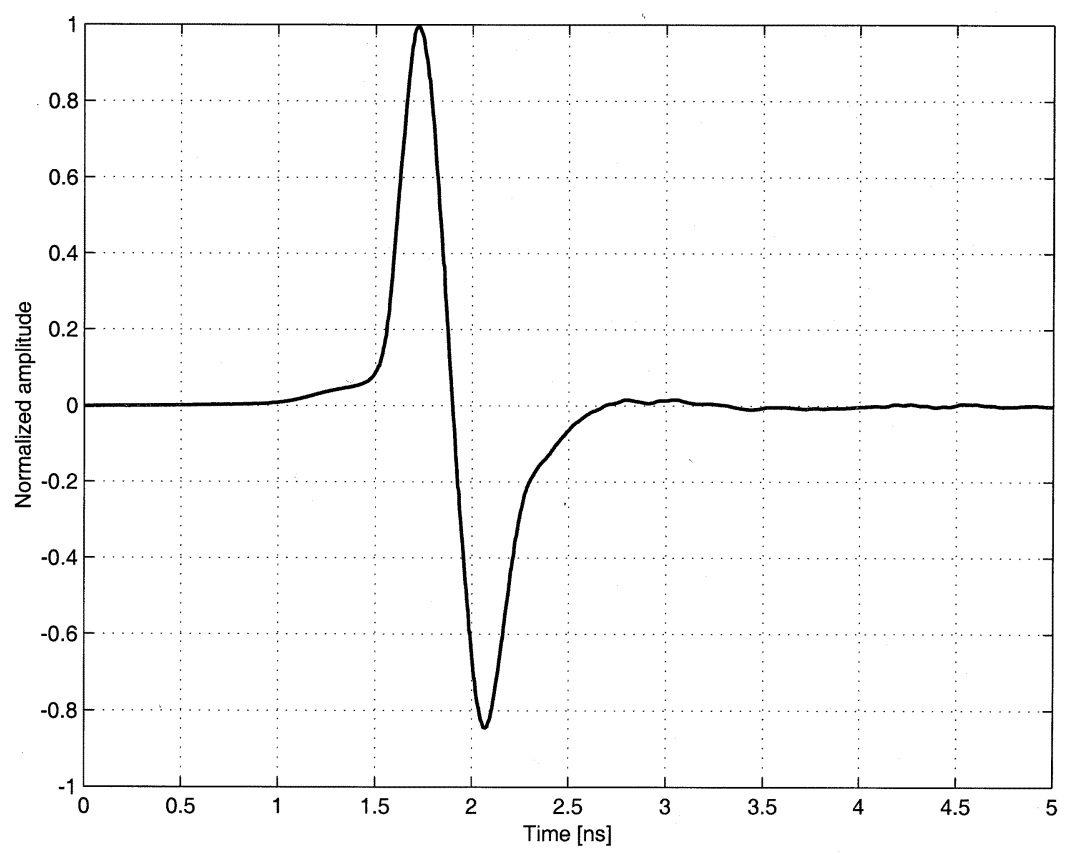

(a)

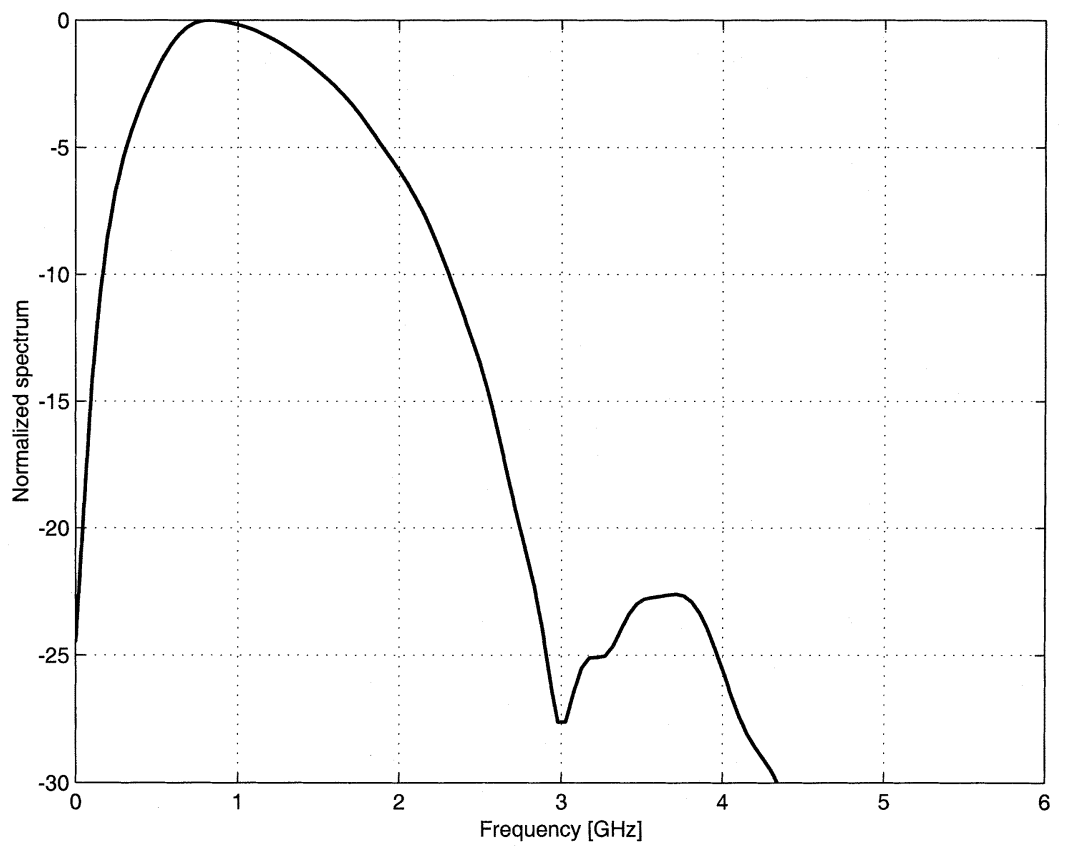

(b)

Fig. 8. Output signal of the $0.8 \mathrm{~ns}$ generator. (a) Waveform of the signal and (b) its frequency spectrum.

surface current in the antenna apex is shown in Fig. 4. It can be seen that in the antenna apex the first reflection arrives at approximately $3.5 \mathrm{~ns}$ after the excitation. This reflection is caused by the reflection from the antenna aperture. Absence of other reflections justifies the chosen flair profile. From values of the voltage in the feeding cell and the current in the antenna apex we derived the antenna input impedance [17]. The current due to the reflection from the aperture is not taken into account in the determination of the input impedance. The frequency dependence of the input impedance is presented in Fig. 5. The antenna impedance remains almost constant in the operational frequency band of the antenna and varies slightly from $62 \Omega$ to $52 \Omega$, while the relatively small reactance slowly increases with frequency.

Radiation into the ground is the most important specification of GPR antennas. The radiated waveform in the near zone within the ground with dielectric permittivity 3.5 and conductivity $0.005 \mathrm{~S} / \mathrm{m}$ is shown in Fig. 6. The waveform is the same as that by radiation into air, while the magnitude of the transient pulse is increased by 1.44 times. Preservation of the radiated waveform has been observed for different types of soil, which shows 


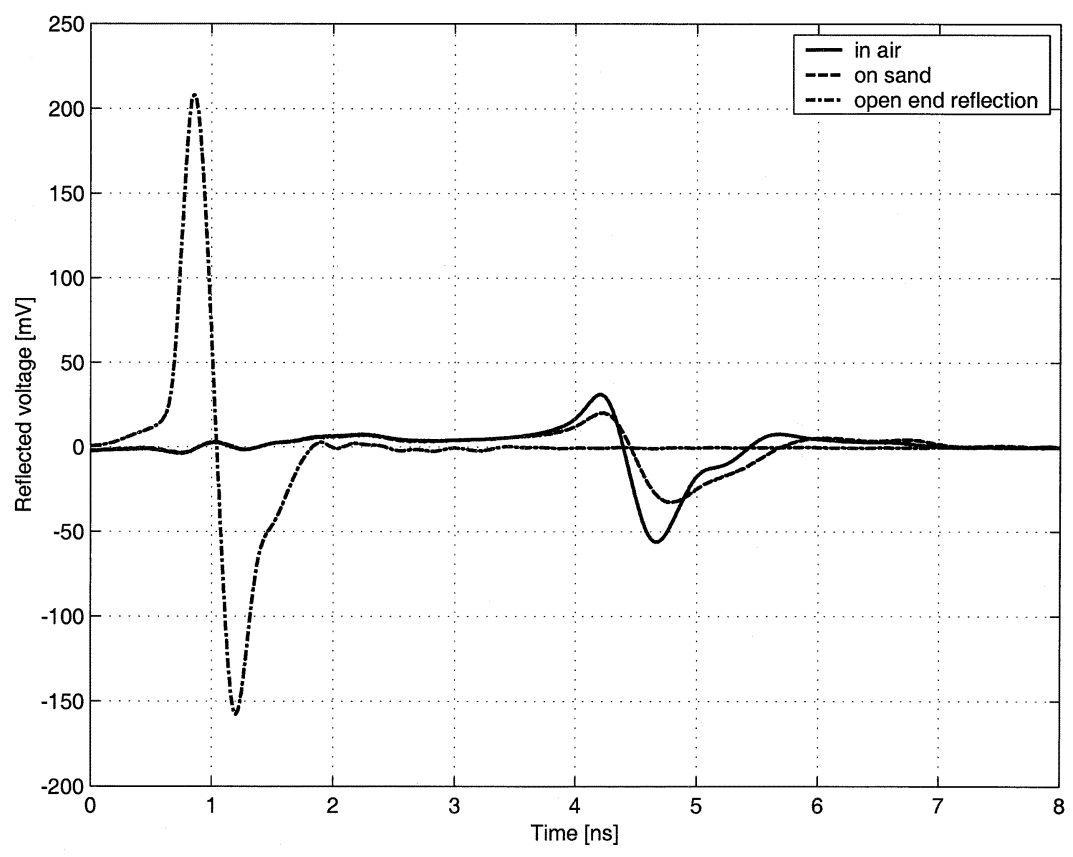

Fig. 9. Measured reflection from the antenna.

stability of the antenna performance in different environmental conditions. The antenna elevation above the ground does not change the waveform of the main pulse either, while a lossy ground in contact with the antenna aperture causes a substantial decrease of the reflection from the aperture and late-time antenna ringing. When the antenna touches the ground, the reflection from the antenna aperture decreases with respect to the radiation into free space. This can be seen in Fig. 7, where the electric field within the wedge is plotted. In this figure the signal between $1 \mathrm{~ns}$ and $2.2 \mathrm{~ns}$ corresponds to the initial radiation, which propagates from the feed point to the aperture. Placing the antenna on the ground does not change the magnitude of this signal. The signal between $3 \mathrm{~ns}$ and $4 \mathrm{~ns}$ is due to the aperture reflection. The magnitude of the last signal is decreased when the antenna is placed on the ground. This proves a better matching of the antenna to the ground than to the air.

\section{EXPERIMENTAL VERIFICATION}

Several experimental models of the dielectric wedge antenna have been built. The dielectric wedges have been made from a single piece of artificial dielectric. The latter represents an epoxy matrix in which titanium dioxide granules are homogeneously distributed. By varying the concentration of the granules, one can obtain different dielectric permittivity values. Ohmic losses of such a material are always very low. Several wedges with different dielectric permittivities (varying from 3 to 7) have been produced. Some measurement results for the dielectric wedge antenna with $\varepsilon=3.5$ and ohmic losses less than $0.001 \mathrm{~S} / \mathrm{m}$ are presented below.

A monocycle generator with a pulse duration of $0.8 \mathrm{~ns}$ has been used in the measurements. The waveform of the generator output is presented in Fig. 8(a), while the normalized spectrum of the pulse is presented in Fig. 8(b). The antenna has been excited via a $50-\Omega$ coaxial line without balun. Unbalanced feeding of the antenna did not cause any problems except for a common-mode current, reflected from the antenna and propagated outside the coaxial line. This current was absorbed by a soft absorbing material applied around the coaxial line.

The transient reflection from the experimental antenna is presented in Fig. 9. The signal, shown in Fig. 9 at the time interval between $0.5 \mathrm{~ns}$ and $1.5 \mathrm{~ns}$, is the reflection from the feed point. The waveform of this signal exactly reproduces the waveform of the incident pulse, which means that the input impedance of the antenna is close to purely real one. From the value of the reflected signal we estimated that the input impedance of the antenna is equal to $54 \Omega$, which is in a good agreement with the theoretical prediction. Reflection from the aperture takes place at the time interval between $4 \mathrm{~ns}$ and $6 \mathrm{~ns}$. It can be seen that for the ground-based antenna the reflection from the aperture is considerably less than the reflection from the aperture-air interface. So the matching to the ground is indeed better than that to air. For reference purposes, Fig. 9 also contains a curve that corresponds to the reflection from the open end of the coaxial feeding line. This signal is shown delayed in time to arrive approximately at the same time as the reflection from the antenna feed point. Compared to the TWIT77 antenna without resistive terminated pad [13] the dielectric wedge antenna has a reflection from the feed point that is 11-times smaller and a reflection from the aperture that is two-times smaller.

The radiated waveform measured along the main axis of the antenna is shown in Fig. 10. Radiation from the antenna has been measured by means of a home-made ultra-wideband sensor [18], which enables retrieval of the waveform and the absolute value of the incident electric field. The radiated pulse consists of contributions from several waves. First, an air-wave contribution is observed at the time interval from $2.0 \mathrm{~ns}$ to $2.8 \mathrm{~ns}$. This wave propagates outside the dielectric wedge and thus arrives earlier at the observation point. Second, 


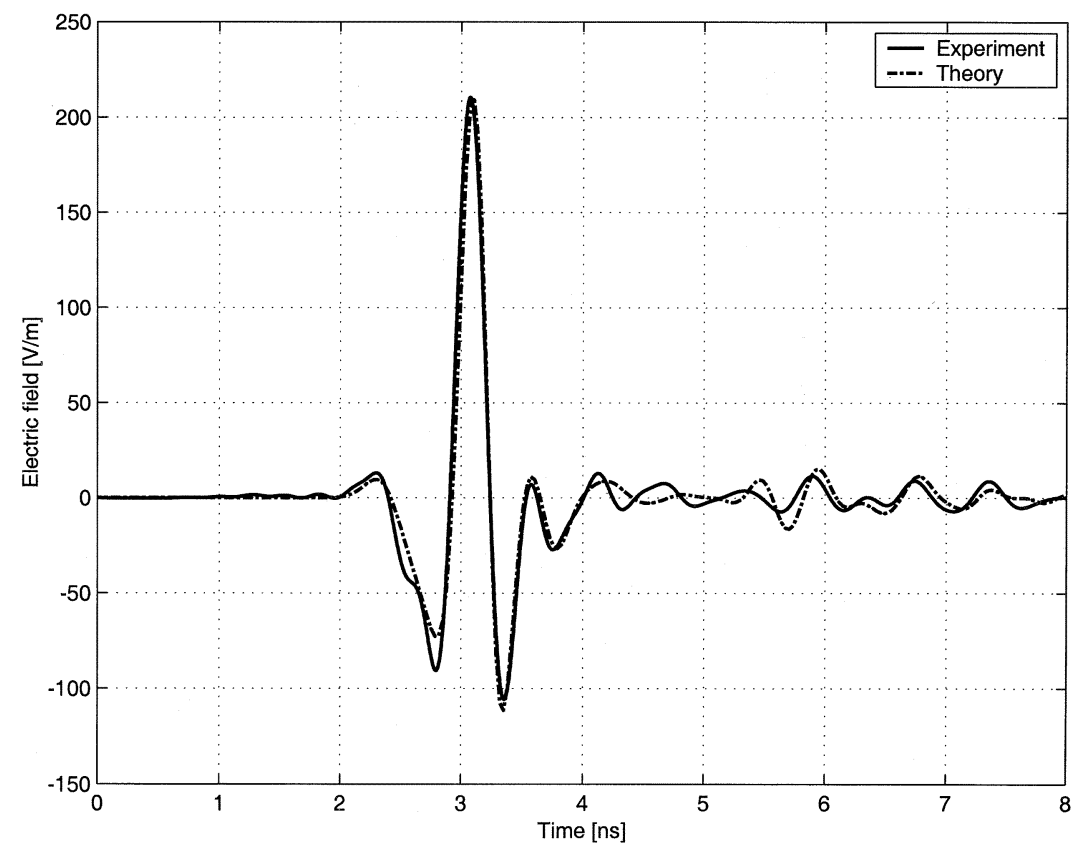

Fig. 10. Radiation from the dielectric wedge antenna into air (solid line: experimental data; dashed line: FDTD simulation). The observation point is situated 39 $\mathrm{cm}$ from the aperture on the main axis of the antenna.

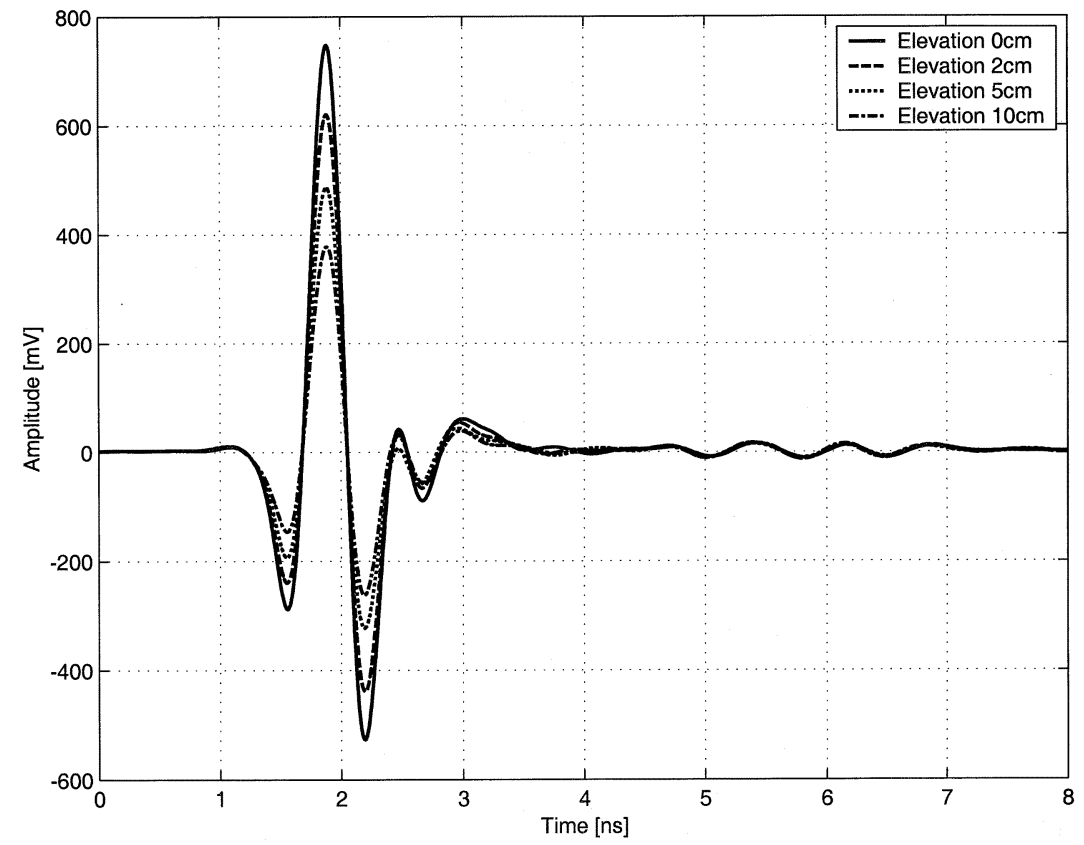

Fig. 11. Elevation profile of the dielectric wedge antenna above sandy ground. The EM field sensor is buried $16 \mathrm{~cm}$ beneath the sand surface.

the main pulse arrives at the interval from $2.6 \mathrm{~ns}$ till $3.4 \mathrm{~ns}$, and is similar to the derivative of the exciting voltage. Short time ringing due to the diffraction on the aperture is observed from $3.4 \mathrm{~ns}$ to $4.3 \mathrm{~ns}$. Finally, at times from $5.5 \mathrm{~ns}$ to $7 \mathrm{~ns}$ the re-radiated reflection from the aperture can be observed. A good agreement between theoretical and experimental results can be seen throughout except for the late time ringing (between $5.2 \mathrm{~ns}$ and $6.2 \mathrm{~ns}$ ). This is due to the discrepancy between the theoretical model in that the feeding line is modeled as a $50-\Omega$ resistor and the experimental model, in which a part of the wave reflected from the aperture propagates along the feeding line as a common mode current and transfers energy away from the antenna. Since the latter phenomenon is neglected in the theoretical model, the comparison with the experiment shows its importance.

The radiation of the dielectric wedge antenna into the ground has been measured in GPR antenna test ranges, developed in IRCTR [19]. Two different test ranges have been used: indoor test range with dry sand (Figs. 11 and 14) and outdoor test range with slightly wet sand (Figs. 12 and 13). The signatures (re- 


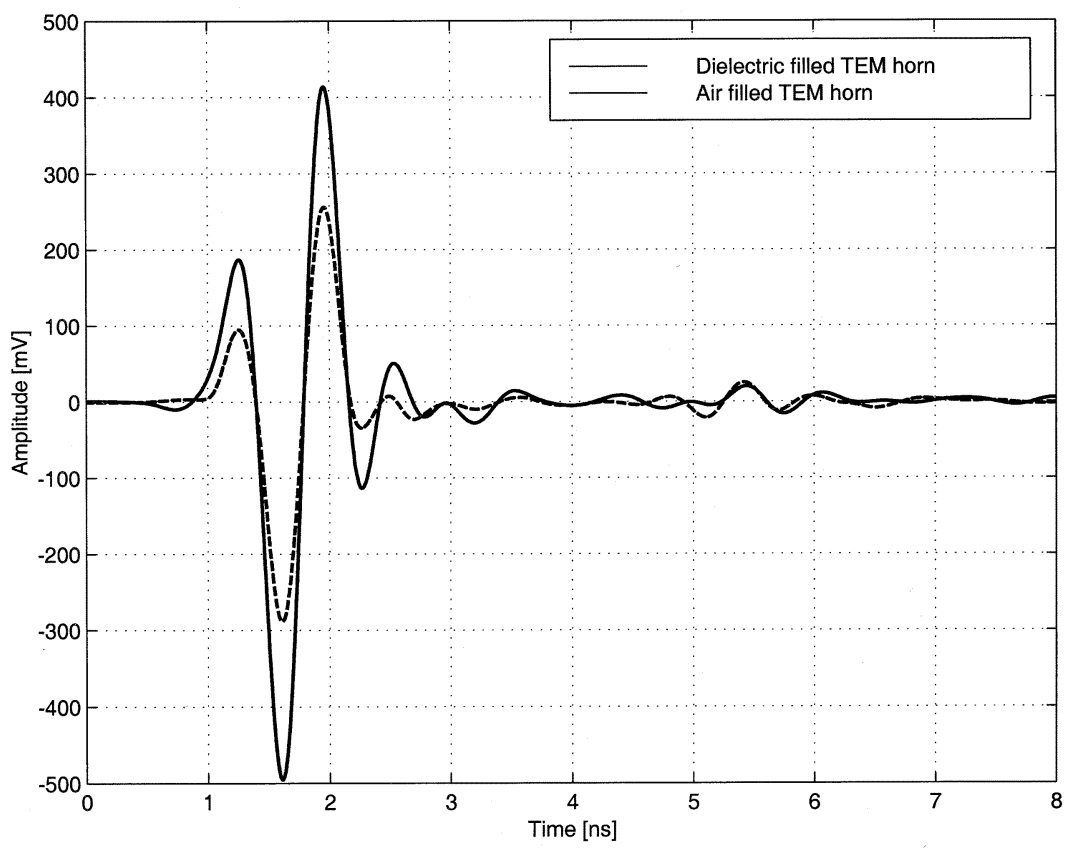

Fig. 12. The sensor's response in the sandy ground to antenna radiation (solid line: dielectric wedge antenna; dashed line: air-filled tapered TEM horn). The EM field sensor is buried $17.5 \mathrm{~cm}$ beneath the sand surface.

sponses of an EM sensor buried in the sand) of the dielectric wedge antenna for different elevations above the ground are presented in Fig. 11. In order to detect changes in waveforms we shifted all signatures in time to compensate for the time delay due to propagation. It can be observed that the main part of the signal as well as the early ringing remains stable for any antenna elevation above the ground. This proves the stability of the antenna performance for different environments. The comparison between radiation into the ground of an "air-filled" TEM horn antenna [12] and of the dielectric wedge antenna is presented in Fig. 12. Both antennas have been fed with the same generator and measured under the same conditions. It can be seen that the magnitude of the signal radiated by the dielectric wedge antenna is 1.48 times larger than that radiated by the "air-filled" TEM horn. Relative magnitude of the late-time ringing (with respect to the main pulse magnitude) by the dielectric wedge antenna is 2.5 times smaller than that by the "air-filled" TEM horn.

For GPR antennas, one must know the spatial distribution of the maximal value of the radiated signal in the near field during the whole observation time (footprint). Such a footprint (in decibels normalized to the maximal value in copolarization) is presented in Fig. 13. The footprint has been measured at an antenna elevation of $1 \mathrm{~cm}$ above the ground. Because the whole footprint measurement takes a lot of time, only a part has been measured. The lines of equal magnitudes of the $y$ component of the electric field have an elliptical shape with a shorter axis along the $\mathrm{E}$ plane. The ellipse main axes equal $30 \mathrm{~cm}$ and $35 \mathrm{~cm}$ at the level of $-10 \mathrm{~dB}$. In the part of the footprint limited by the level of $-10 \mathrm{~dB}$, the radiated waveform is similar to the waveform measured in the far field. The space-frequency representations of the radiated field in $\mathrm{E}$ plane and in $\mathrm{H}$ plane as measured in the sand are presented in Fig. 14. It can be seen that the footprint size decreases linearly with the increase of frequency. The maximum of the radiated field spectrum is observed at $1.3 \mathrm{GHz}$, which is

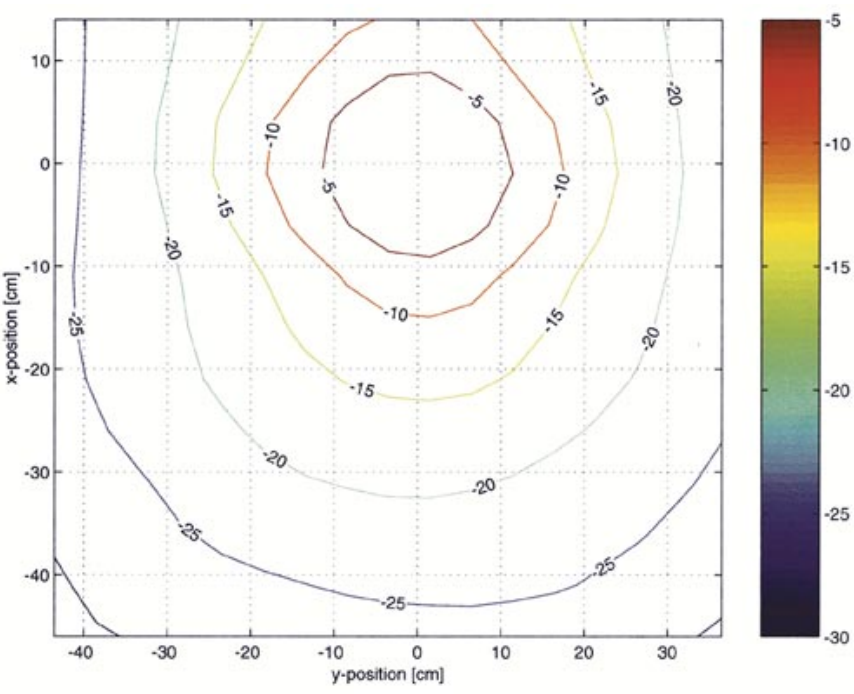

Fig. 13. Antenna footprint (in $\mathrm{dB}$ ) in the sand measured at the depth $17.5 \mathrm{~cm}$. The antenna is elevated $1 \mathrm{~cm}$ above the ground.

higher than that of the generator output. Interference between the air wave and the wedge wave can be seen at the figures.

Finally the coupling between two- identical dielectric wedge antennas has been measured. As we expected in advance, the dielectric filling has decreased coupling between $\mathrm{Tx}$ and $\mathrm{Rx}$ antennas in the antenna system (Fig. 15). Direct coupling from the feed point of the Tx antenna to the feed point of the Rx antenna is observed at the time interval from $1 \mathrm{~ns}$ to $2 \mathrm{~ns}$. Between $2.5 \mathrm{~ns}$ and $7.2 \mathrm{~ns}$, aperture coupling is observed. The duration of the coupling between two dielectric wedge antennas is the same as that of the conventional TEM horns. However, the magnitude of the coupling is 2.2 times smaller and corresponds to the antenna isolation level below $-42 \mathrm{~dB}$ within the feeding pulse bandwidth. 


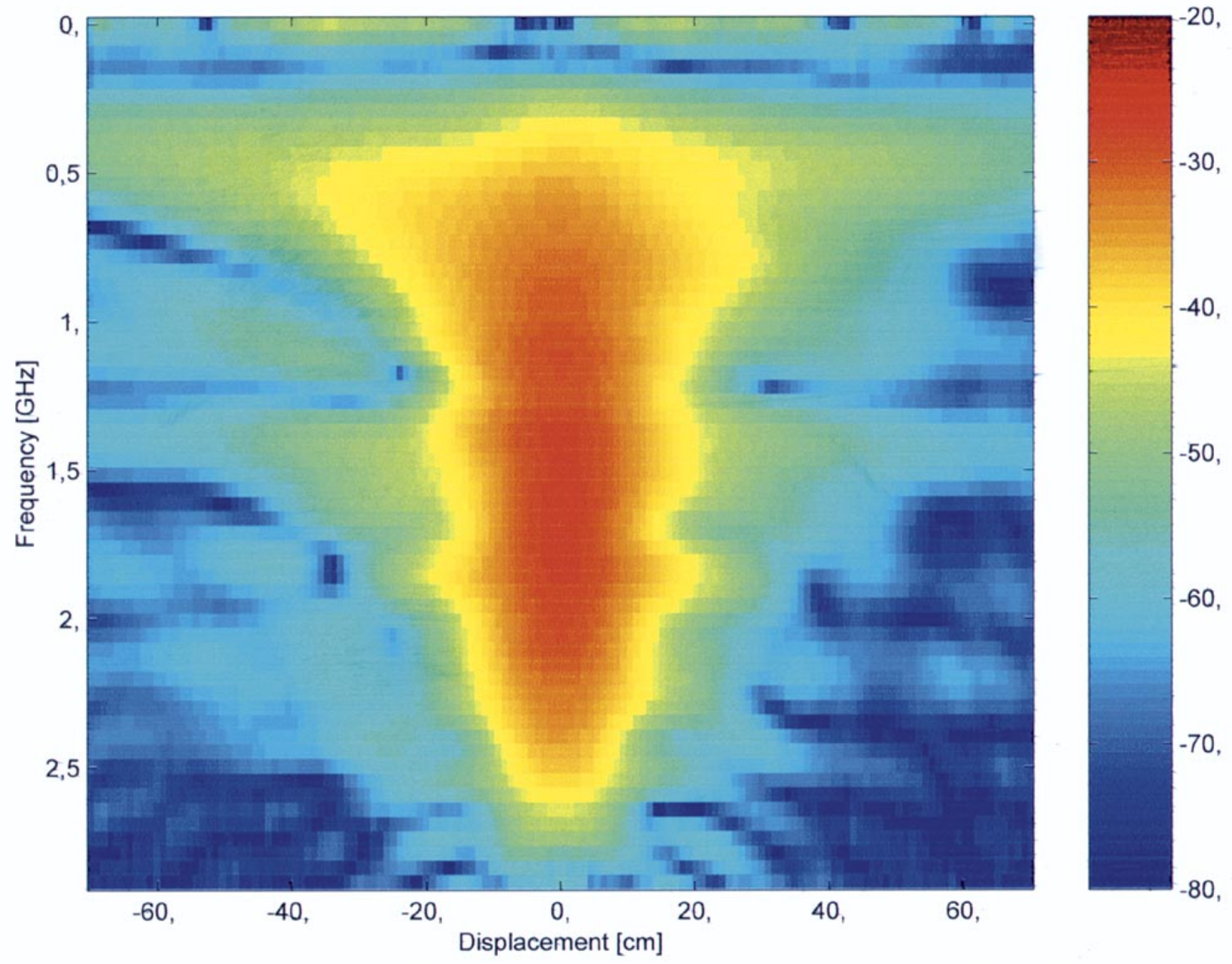

(a)

Fig. 14. Space frequency characterization of the antenna radiation into sand (a) E-plane and (b) H-plane. Spectral content is shown in logarithmic scale. The EM field sensor is buried $16 \mathrm{~cm}$ beneath the sand surface.

\section{DISCUSSIONS AND CONCLUSION}

A new modification of the TEM-horn antenna has been developed for GPR applications. The antenna is based on a dielectric wedge and therefore, was named the dielectric wedge antenna. The metal flairs were tapered to match the antenna to the ground and to reduce the late-time ringing. The size (for a given dielectric permittivity) of the wedge has been optimized to obtain the maximal amplitude of the radiated pulse. The tapering profile has been calculated based on a simple microstrip-line model and has been justified by means of 3-D FDTD simulation. We verified our theoretical design using experimental prototypes. We found experimentally that the dielectric wedge antenna radiates a 1.48 times larger pulse into the sandy halfspace, possesses 2.5 times smaller magnitude of the re-radiated reflection from the aperture, provides 2.2 times smaller coupling in the antenna system, and finally, is approximately two-times smaller than the tapered "air-filled" TEM horn (designed for radiation of same pulses in free space). The dielectric wedge antenna has shown a stable performance for different elevations above the ground and low sensitivity to external EMI.
Despite the tapered flair profile, the reflection from the aperture still causes some late-time ringing of the antenna (about 6\% from the magnitude of the main pulse). There are different possibilities to reduce this late-time ringing (e.g., resistive terminating pads [11], [13], improving the tapering profile or loading the antenna with a lossy dielectric material), however a comparative analysis of these possibilities lies outside the scope of this paper and will be reported elsewhere.

The air wave, which propagates outside the wedge, causes an increase in the duration of the radiated pulse and a decline of the leading edge of the pulse. This wave can be effectively suppressed by dielectric loading of the antenna. Covering both metal flairs with dielectric slabs of the same dielectric permittivity as the dielectric permittivity of the wedge considerably reduces the contribution of the air wave in the intermediate and far zones. The developed FDTD model of the antenna has shown to be a useful tool for further antenna optimization.

The developed antenna can be used successfully for a conventional GPR if the Tx-Rx antennas are in direct contact with the ground. However, in applications such as those for landmine detection, antenna elevation (above the ground) is 


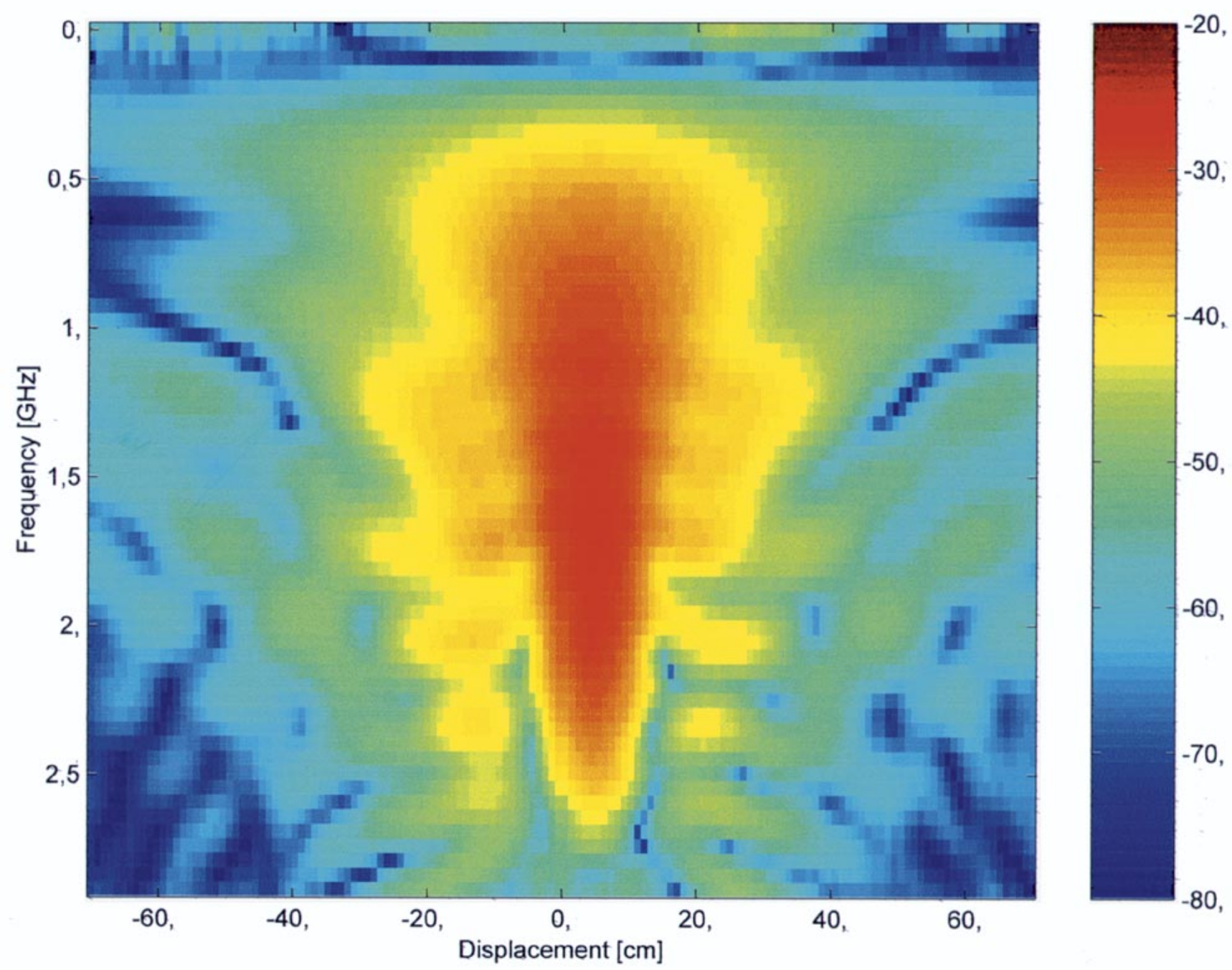

(b)

Fig. 14. (Continued.) Space frequency characterization of the antenna radiation into sand (a) E-plane and (b) H-plane. Spectral content is shown in logarithmic scale. The EM field sensor is buried $16 \mathrm{~cm}$ beneath the sand surface.

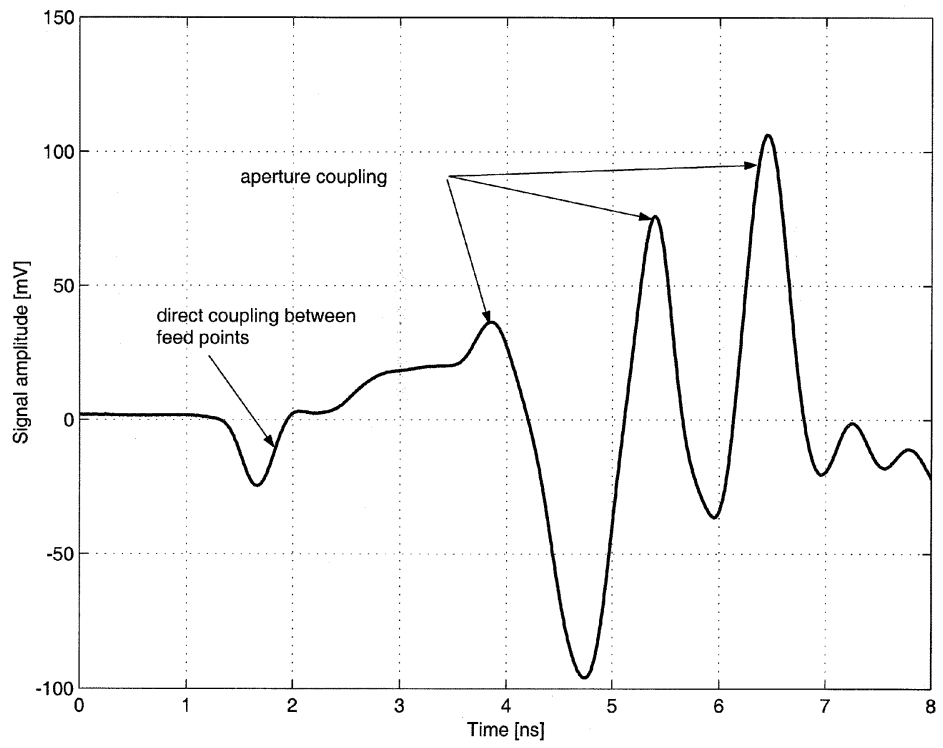

Fig. 15. Coupling in air between two dielectric wedge antennas excited by $0.8 \mathrm{~ns}$ monocycle with $50 \mathrm{~V}$ peak-to-peak amplitude. Separation between antennas is $30 \mathrm{~cm}$. 
necessary. It was found that the developed antenna can be used in this case as well, because the antenna radiates short pulses with relatively small ringing not only into the ground, but also into the air. In such a case a dielectric lens attached to the antenna aperture improves its performance. The dielectric wedge antenna has been used successfully as a transmit antenna in a novel antenna configuration within a GPR system designed for landmine detection [20]. Partly due to this specific antenna configuration, it became possible to produce high-resolution images of surface and subsurface objects, which allowed detection and sometimes visual classification of different anti-tank and anti-personnel mines [21].

\section{ACKNOWLEDGMENT}

The authors would like to thank G. Mur for providing his FDTD code and very friendly support for antenna simulations, G. Smith for helpful discussions on applications of the FDTD method for transient antennas simulation, D. Noon and E. Farr for indepth discussions on the construction and operation of the antenna and the reviewers for their comments and suggestions.

\section{REFERENCES}

[1] D. J. Daniels, Surface-Penetrating Radar. London, U.K.: Inst. Elect. Eng., 1996.

[2] G. Smith, "Directive properties of antennas for transmission into a material halfspace," IEEE Trans. Antennas Propagat., vol. AP-32, no. 3 , pp. 232-246, Mar. 1984.

[3] G. Turner, "Modeling antenna-ground interactions," in Proc. 5th Int. Conf. .Ground-Penetrating Radar, vol. 1, Kitchener, ON, Canada, June 12-16, 1994, pp. 205-221.

[4] M. Cherniakov and L. Donskoi, "Frequency band selection of radars for buried object detection," IEEE Trans. Geosci. Remote Sensing, vol. 37, pp. 838-845, Mar. 1999.

[5] J. D. Young, "Antenna Development for Pipe Locating Radar Systems," The Ohio State University ElectroScience Laboratory, Columbus, $\mathrm{OH}$, $529081-1,1980$.

[6] D. J. Daniels, D. J. Gunton, and H. F. Scott, "Introduction to subsurface radar," Proc. Inst. Elect. Eng. , pt. F, vol. 135, no. 4, pp. 278-321, Aug. 1988.

[7] P. Roimela, S. Salmenkaita, P. Maijala, and T. Saarenketo, "Road analysis-A tool for effective rehabilitation measures for finnish roads," in Proc. 8th Int. Conf. Ground Penetrating Radar, vol. SPIE 4084, D. A. Noon, G. F. Stickley, and D. Longstaff, Eds., 2000, pp. 107-112.

[8] D. J. Daniels, C. Martel, and M. Philippakis, "Progress in GPR for mine detection," in Proc. 8th Int. Conf. Ground Penetrating Radar, vol. SPIE 4084, D. A. Noon, G. F. Stickley, and D. Longstaff, Eds., 2000, pp. 243-248.

[9] C. C. Chen, "A new ground penetrating radar antenna design-The horn-fed bowtie (HFB)," in Proc. 20th Annu. Meeting Symp. Antenna Measurement Techniques Assoc., 1998, pp. 67-74.

[10] B. Scheers, M. Piette, and A. V. Vorst, "Development of dielectric-filled TEM horn antennas for UWB GPR," in Proc. Millennium Conf. Antennas Propagat., Davos, Switzerland, Apr. 9-14, 2000.

[11] K. L. Schlager, G. S. Smith, and J. G. Maloney, "TEM horn antenna for pulse radiation: An improved design," Microwave Opt.Technol. Lett., vol. 12, pp. 86-90, June 1996.

[12] R. V. de Jongh, A. G. Yarovoy, L. P. Ligthart, I. V. Kaploun, and A. D. Schukin, "Design and analysis of new GPR antenna concepts," in Proc. 7th Int. Conf. Ground Penetrating Radar, vol. 1, Lawrence, Kansas, May 27-30, 1998, pp. 81-86.

[13] E. A. Theodorou, M. R. Gorman, P. R. Rigg, and F. N. Kong, "Broadband pulse-optimized antenna," Proc. Inst. Elect. Eng. , pt. H, vol. 128, no. 3 , pp. 124-130, June 1981.
[14] H. A. Wheeler, "Transmission-line properties of parallel strips separated by a dielectric sheet," Trans. IEEE Microwave Theory Tech., vol. MTT-13, pp. 172-185, Mar. 1965.

[15] K. L. Schlager, G. S. Smith, and J. G. Maloney, "Optimization of bow-tie antenna for pulse radiation," IEEE Trans. Antennas Propagat., vol. 42, pp. 975-982, July 1994.

[16] G. Mur, "Total-field absorbing boundary conditions for the time-domain electromagnetic field equations," IEEE Trans. Electromagn.Compat., vol. 40, pp. 100-102, May 1998.

[17] R. Luebbers and K. Kunz, "Finite difference time domain calculations of antenna mutual coupling," IEEE Trans. Electromagn. Compat., vol. 34, pp. 357-359, Aug. 1992.

[18] A. G. Yarovoy, R. V. de Jongh, and L. P. Ligthart, "Ultra-wideband sensor for electromagnetic field measurements in time domain," Electron. Lett., vol. 36, no. 20, pp. 1679-1680, Sept. 2000.

[19] R. V. de Jongh, A. G. Yarovoy, and L. P. Ligthart, "Experimental set-up for measurement of GPR antenna radiation patterns," in Proc. 28th European Microwave Conf., vol. 2, Amsterdam, Oct. 6-8, 1998, pp. 539-543.

[20] A. G. Yarovoy, P. van Genderen, and L. P. Ligthart, "Ground penetrating impulse radar for landmine detection," in Proc. 8th 2000 Int. Conf. Ground Penetrating Radar, , vol. SPIE 4084, 2000, pp. 856-860.

[21] J. Groenenboom and A. G. Yarovoy, "Data processing for landmine detection dedicated GPR," in Proc. 8th 2000 Int. Conf. Ground Penetrating Radar, vol. SPIE 4084, 2000, pp. 867-871.

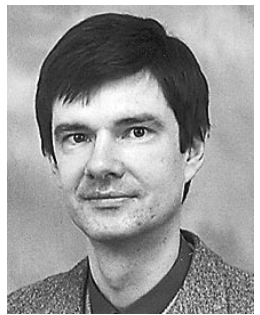

Alexander G. Yarovoy (M'95) received the Diploma degree (Hons.) in radiophysics and electronics, the Cand. Phys. \& Math. Sci. and the Dr. Phys. \& Math. Sci. degrees in radiophysics from from Kharkov State University, Ukraine, in 1984, 1987, and 1994, respectively.

In 1987, he joined the Department of Radiophysics, Kharkov State University as a Researcher and in 1997 became a Professor. From 1994 to 1996, he was with the Technical University of Ilmenau, Germany as a Visiting Researcher. Since 1999, he had been with the International Research Centre for Telecommunications-Transmission and Radar (IRCTR) at the Delft University of Technology, The Netherlands, where he coordinates all GPR-related projects. His main research interests are in ultrawide-band electromagnetics, wave scattering from statistically rough surfaces and penetrable obstacles and computational methods in electromagnetics.

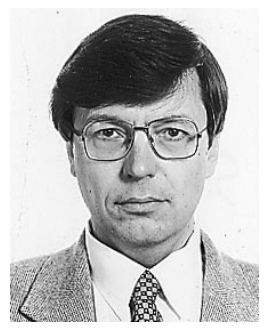

Alexander D. Schukin graduated from the Moscow Power Engineering Institute in 1974.

Currently, he is with the Academician A. L. Mints Radiotechnical Institute, Moscow, as a Senior Engineer in antenna technology working on the design of UHF systems. His research interests includetime domain and frequency domain antenna measurements and numerical methods for parameter optimization.

Igor V. Kaploun was born on August 9, 1948. He received the graduation degree from Moscow Physical-Technical University, and the Ph.D. degree from Moscow Device-Building Institute in 1972 and 1976, respectively.

He is currently with the Academician A. L. Mints Radiotechnical Institute, Moscow, as the Head of the Antenna Department. His research interests include UHF antennas and wave propagation. 


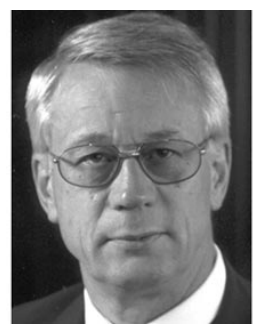

Leo P. Ligthart (M'94-SM'95-F'02) was born in Rotterdam, The Netherlands, on September 15, 1946. He received the Engineer's degree (cum laude) and the Doctor of Technology degree from the Delft University of Technology, The Netherlands, in 1969 and 1985, respectively. He received the Ph.D degree (honoris causa) from Moscow State Technical University of Civil Aviation and from the Tomsk State University of Control Systems and Radioelectronics in 1999 and 2001, respectively.

Since 1992, he has held the Chair of Microwave Transmission, Radar, and Remote Sensing, in the Department of Information Technology and Systems, Delft University of Technology. In 1994, he became Director of the International Research Center for Telecommunications-Transmission and Radar. His research interests include antennas and propagation, radar, and remote sensing, satellite, mobile, and radio communications. 